\title{
Contribution of Computed Tomographic Angiography to Pretreatment Planning of Radio-embolization of Liver Tumors
}

\author{
HYNEK MIRKA $^{1,2}$, PETR DURAS $^{1}$, JAN BAXA $^{1}$, EVA KORCAKOVA $^{1,2}$ and JIRI FERDA ${ }^{1}$ \\ ${ }^{1}$ Department of Imaging Methods, Faculty of Medicine in Pilsen, \\ Charles University in Prague and Teaching Hospital, Pilsen, Czech Republic; \\ ${ }^{2}$ Biomedical Centre, Faculty of Medicine in Pilsen, Charles University in Prague, Pilsen, Czech Republic
}

\begin{abstract}
Transarterial radio-embolization (TARE) using yttrium-90 microspheres is a promising method based on the brachytherapeutic effect of radionuclide with beta-minus decay dissolved in solid microparticles applied directly to tumor-supplying arteries. This treatment is complex, as well as logistically and technically extremely demanding and must be planned in detail. The visualization of the vascular supply of the liver and the possible parasitic supply of the tumor is essential not only for indication of the procedure and correct identification of the arteries to which the microspheres will be applied, but also for prevention of nontarget deposition of radioactive material. This review addresses the use of computed tomographic angiography in the preparatory phase of TARE.
\end{abstract}

Locoregional treatment is currently an important part of comprehensive care for patients with liver tumors. Specifically, it is indicated in patients with primary and secondary malignancies who are not suitable candidates for liver resection or transplantation. Standard methods used in this area are thermal ablation (radiofrequency ablation, microwave ablation or cryo-ablation) and intra-arterial treatment (TACE) (1). Transarterial radio-embolization (TARE) using yttrium-90containing glass or resin microspheres is a promising new method based on the brachytherapeutic effect of radionuclide

This article is freely accessible online.

Correspondence to: Associate Professor Hynek Mírka, MD, Ph.D., Department of Radiology, Medical School and Teaching Hospital Pilsen, Charles University in Prague, Alej Svobody 80, 30460 Plzen, Czech Republic. Tel: +420 377103456, Fax: +420 377103438, e-mail:mirka@fnplzen.cz

Key Words: Radio-embolization, yttrium-90, liver tumor, computed tomography, angiography, review. particles applied directly to the tumor-supplying arteries (2). This treatment is complex, as well as logistically and technically extremely demanding, and must be planned in detail. Therefore, the anatomical and functional parameters of the liver and its circulation are assessed in a multimodal manner using radiological and nuclear medicine methods. The visualization of the vascular supply of the liver and the possible parasitic supply of the tumor is essential, not only for indication of the procedure and correct identification of the arteries to which the microparticles will be applied, but also for prevention of non-target deposition of radioactive material (3). This review addresses the use of imaging methods in mapping of the liver arterial anatomy in the preparatory phase of TARE.

\section{Arterial Supply of the Liver and its Importance in TARE}

The complex embryonal development of the hepatobiliary system predisposes this area to having a highly variable arterial anatomy (4). A classic arrangement with two hepatic arteries arising from the proper hepatic artery, which is a branch of the common hepatic artery arising from the truncus coeliacus, occurs in 50-61\% of individuals (5-7). In other cases, the arteries are replaced, or accessory arteries are found (Figure 1). According to Michels' classification, 10 types of arterial variants exist (Table I) (8). The arteries that supply the surrounding organs can also originate from hepatic arteries. For example, the right gastric artery, accessory left hepatic artery, lower diaphragmatic artery and umbilical artery may be branches of the left hepatic artery (9). An examination of liver segment IV should be focused on the possible presence of the middle hepatic artery, which may be a branch of the right hepatic artery or arise directly from the proper hepatic artery as a trifurcation with the end branches of the right or left hepatic artery passing on the other side. Moreover, mapping the distance of normal arterial branches from the point of microsphere application is also important, 
Table I. Anatomic variants of hepatic arteries (8).

\begin{tabular}{lcc}
\hline Michels type & Description & Occurrence \\
\hline I & Normal anatomy & $55-61 \%$ \\
II & Replaced LHA & $3-10 \%$ \\
III & Replaced RHA & $8-11 \%$ \\
IV & Replaced RHA and LHA & $1 \%$ \\
V & Accessory LHA & $8-11 \%$ \\
VI & Accessory RHA & $1.5-7 \%$ \\
VII & Accessory RHA and LHA & $1 \%$ \\
VIII & Replaced RHA and accessory & \\
& LHA or accessory RHA & \\
& and replaced LHA & $2.5 \%$ \\
IX & CHA replaced to SMA & $2-4.5 \%$ \\
X & CHA replaced to LGA & $0.5 \%$ \\
Unclassified & CHA arising from aorta & $2 \%$ \\
& Double hepatic artery Replaced PHA & $4 \%$ \\
& Others & $<0.5 \%$ \\
\hline
\end{tabular}

LHA: Left hepatic artery; RHA: right hepatic artery; CHA: common hepatic artery; SMA: superior mesenteric artery; LGA: left gastric artery; PHA: proper hepatic artery.

especially for the gastroduodenal and cystic arteries (10). Specifically, up to $17 \%$ of tumors located on the liver surface have a parasitic arterial supply (Table II) (11).

Non-target radiopharmaceutical deposition may cause serious complications, such as cholecystitis, gastrointestinal ulceration or bleeding, gastritis, duodenitis, pancreatitis, hepatitis, supra-umbilical dermatitis and radiation pneumonitis (12-14). Therefore, all patients undergo protective embolization of the gastroduodenal artery, left gastric artery, cystic artery or other arteries which may allow the non-target deposition of microspheres $(15,16)$. To ensure the redistribution of blood in the liver, individual branches supplying liver tissue may be embolized in some patients. Other adverse factors that increase the probability of embolization in non-target areas are stenoses and the slowing of flow in the hepatic arteries. Proper catheter positioning based on the knowledge of the liver arterial architecture is essential in order to perform the evaluation of liver perfusion and assessment of hepatopulmonary fraction deposition of microspheres before TARE. Hepatopulmonary shunting is evaluated with single photon-emission computed tomography/computed tomography (SPECT/CT) using technetium-99 m-labelled human albumin macro-aggregate in order to assess the potential radiation injury, occurring when the arteriovenous shunting exceeds more than one-tenth of the applied activity.

Imaging of portal venous system is also very important in the planning of TARE. Portal vein thrombosis with the absence of hepatopetal flow increases the likelihood of ischemic involvement of the liver and is a contraindication for TARE using resin microspheres. The procedure with glass microspheres can be successfully performed in this condition (17).
Table II. Extrahepatic collaterals supplying liver tumors (9).

\begin{tabular}{ll}
\hline Location of the tumor & Possible sources of parasitic arteries \\
\hline Bare area of the liver & Right phrenic, right adrenal artery \\
Superior-anterior part & Right internal mammary artery \\
Exophytic toward kidney & Right renal, right adrenal artery \\
Any periotoneal surface & Omental branches of RGA \\
Near chest wall & Lower intercostal artery \\
Left lateral segment & Left gastric artery \\
Near colon & Colic branches of SMA \\
Gallbladder fossa & Gastic artery \\
\hline
\end{tabular}

RGA: Right gastric artery; SMA: superior mesenteric artery.

\section{Methods of Liver Artery Imaging}

Based on the above, the Imaging of liver artery variants and the parasitic supply is necessary in order to optimize the effect of TARE. To this end, the gold standard method is digital subtraction angiography (DSA). DSA consists of the non-selective imaging of the abdominal aorta, the selective imaging of the coeliac axis and superior mesenteric artery, and the super-selective imaging of all arteries that supply the hepatic parenchyma. In addition to the right and left hepatic arteries, the cystic artery, supraduodenal, retroduodenal, falciform, left gastric accessory, and right and left inferior phrenic arteries should also be located. Angiography may be supplemented with cone-beam $\mathrm{CT}$ which, contrary to multidetector CT, uses a two-dimensional detector. From the data obtained, three-dimensional images showing not only the anatomy of the arteries but also the tumor tissue can be reconstructed. Cone-beam CT use in hepatic interventional radiology thus enables more selective catheterization and increased treatment efficacy. The diagnostic part of the procedure may be followed by protective embolization using metallic spirals or tissue glue of arteries that might allow the non-target deposition of microspheres.

Moreover, CT or positron-emission tomography (PET)/CT images of the liver including a series of thin slices in the arterial and venous phase are a common part of liver tumor staging. Specifically, performing the CT scan on a multidetector scanner (optimally with 64 or more detector rows) allows a fairly detailed assessment of vascularity, but hepatic artery imaging can also be performed using magnetic resonance imaging (MRI) (18).

\section{Technique of CT Angiography (CTA)}

CT images of the liver are obtained after the injection of contrast medium (CM) in the arterial, venous and, optionally, equilibrium phases. The volume of $\mathrm{CM}$ should correspond to 500-600 mgI per kilogram of body weight, and the iodine flux should be in the range of $1.6-2.0 \mathrm{~g} / \mathrm{s}$. CM injection is 

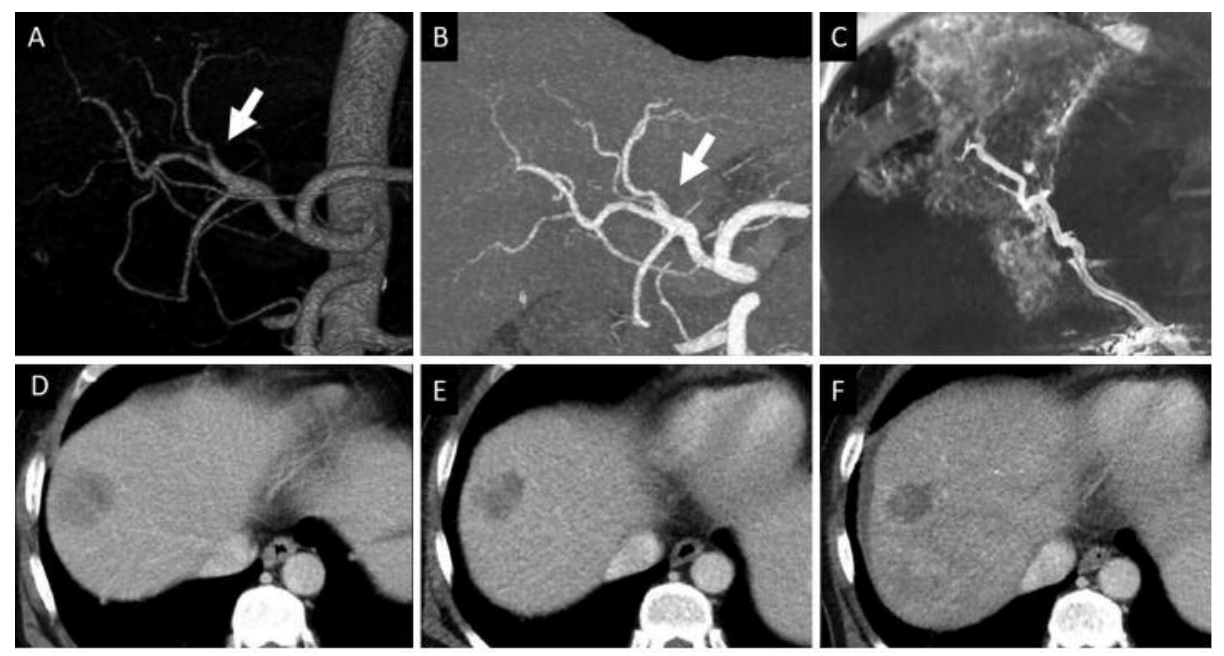

Figure 1. Computed tomography angiography (CTA) of the liver showing the intrahepatic segmentation of the hepatic artery. A trifurcation of the hepatic artery (arrow) is visible. The supplementary artery supplies the gall bladder and part of the metastasis. A: CTA, volume-rendered 3D reconstruction. B: CTA, maximum intensity projection of hepatic arteries. C: Catheter-based perfusion image from cone-beam CT. D: Axial CT image of liver metastasis before transarterial radio-embolisation (TARE). E: Axial CT image 6 weeks after TARE. F: Axial CT image 6 months after TARE, with visible size reduction and decrease in density representing necrosis.

followed by a saline flush to ensure high-quality visualization of both the arterial and venous system, the liver parenchyma, and possible focal lesions (19). In order to increase the contrast between the vessels and structures of the gastrointestinal tract, the per-oral application of water or mannitol water solution is advisable. Moreover, if the examination is performed only to map the liver artery, the volume of CM can be reduced by approximately $20 \%$ (20).

The early arterial phase (15-20 s after the start of CM injection or immediately after reaching a threshold of 100 $\mathrm{HU}$ in the aorta) is recommended for arterial imaging. At this stage, the concentration of CM is sufficient in the arteries but not in the portal vein and hypervascular lesions, which are not yet enhanced. Therefore, the signal-to-noise ratio is higher between the vessels and surrounding structures, which simplifies the visualization of small arteries. However, liver tumors should be imaged in the late arterial phase (approximately 35-40 s after the start of the contrast medium injection or 15-20 s after reaching a threshold of $100 \mathrm{HU}$ in the aorta). At this phase, the portal vein and hypervascular liver lesions are enhanced, which results in a lower signalto-noise ratio. However, in a study to compare the benefits of early and late arterial phase examinations of liver arterial variants, Van den Hoven et al. showed that liver arterial imaging did not produce significantly worse results in the late arterial phase than the early arterial phase (21).

In non-obese patients, the low-voltage (80-100 kV) technique is preferred in because it increases the CM contrast and consequently increases the resolution of blood vessels and hypervascular focal lesions at a lower radiation dose (22). Depending on the voltage drop, the current must be increased to optimize image quality, and the ratio of contrast to noise is not significantly reduced under these conditions (23). To optimize the exposure parameters, automatic $\mathrm{kVp}$ setting and automatic off-line and on-line current modulation systems are currently used. Alternatively, image noise and radiation load can also be reduced using iterative reconstructions.

Scanning on multi-detector devices is performed using submillimetric collimation (typically $0.5-0.75 \mathrm{~mm}$ ), which ensures an isotropic data field that allows multiplanar reformations and three-dimensional reconstructions in any plane without a loss of resolution. To increase spatial resolution, some authors recommend the use of the edgeenhancement reconstruction algorithm (24). Thin axial sections and multiplanar reformations or maximum intensity projection reconstructions are used for evaluation (25).

\section{The Importance of CTA}

CTA provides sufficient time and spatial resolution for replacing diagnostic angiography in clinical practice. Optimally performed examinations can detect arterial branches with a diameter of $1 \mathrm{~mm}$, and visualizing the hepatic arteries with proximal widths between $2-3 \mathrm{~mm}$ is usually sufficient $(26,27)$. Comparative studies of the accuracy of CTA and other methods (DSA, cone-beam CT) indicate that DSA is more accurate when detecting liver 
arterial anomalies, especially in the area of the right hepatic artery (detectable at 65\% CTA). However, the authors of these studies also agree that CTA accurately assesses the supply of the fourth hepatic segment (up to $36 \%$ higher detecability than with DSA) $(21,28,29)$. Similarly, good results were obtained in the detection of the falciform artery - Burgman et al. detected this artery in 4.5 as many patients when using CTA compared with DSA (52.3 vs. $11.9 \%$ ) (29). MRI, alone or as a part of PET/MRI, is another commonly used method for detection, differential diagnosis and staging of liver tumors. As with CT, liver artery anatomy can be evaluated in images obtained in the arterial phase. However, this method is less accurate than CTA; one study showed that the reliability of MRI was lower than that of CTA, especially in the area of the left hepatic artery (18).

Despite the limitations associated with CTA, it can be considered a suitable method for planning the catheterization procedure. Specifically, it accelerates and consequently reduces the radiation load and the amount of CM required, which is beneficial, especially for patients with impaired renal function $(10,21,28)$.

\section{Acknowledgements}

This article was supported by the project of the Ministry of HealthConceptual Development of Research Institutions 00669806 - FN Plzen, by the project of the Charles University Prague Progress Q39 and by the project CZ.1.05/2.1.00/03.0076 from the European Regional Development Fund.

\section{References}

1 Lencioni R: Loco-regional treatment of hepatocellular carcinoma. Hepatology 52: 762-773, 2010.

2 Sacco R, Mismas V, Marceglia S, Romano A, Giacomelli L, Bertini M, Federici G, Metrangolo S, Parisi G, Tumino E, Bresci G, Corti A, Tredici M, Piccinno M, Giorgi L, Bartolozzi C and Bargellini I: Transarterial radioembolization for hepatocellular carcinoma: An update and perspectives. World J Gastroenterol 21: 6518-6525, 2015.

3 Riaz A, Awais R and Salem R: Side-effects of yttrium-90 radioembolization. Front Oncol 4: 198, 2014.

4 Liu DM, Salem R, Bui JT, Courtney A, Barakat O, Sergie Z, Atassi B, Barrett K, Gowland P, Oman B, Lewandowski RJ, Gates VL, Thurston KG and Wong CY: Angiographic considerations in patients undergoing liver-directed therapy. J Vasc Interv Radiol 16: 911-935, 2005.

5 Lee AJ, Gomes AS, Liu DM, Kee ST, Loh CT and McWilliams JP: The road less traveled: importance of the lesser branches of the celiac axis in liver embolotherapy. Radiographics 32: 11211132, 2012.

6 Ugurel MS, Battal B, Bozlar U, Nural MS, Tasar M, Ors F, Saglam M and Karademir I: Anatomical variations of hepatic arterial system, coeliac trunk and renal arteries: an analysis with multidetector CT angiography. Br J Radiol 83: 661-667, 2010.
7 Covey AM, Brody LA, Maluccio MA, Getrajdman GI and Brown KT: Variant hepatic arterial anatomy revisited: Digital subtraction angiography performed in 600 patients. Radiology 224: 542-547, 2002.

8 Michels NA: Newer anatomy of the liver and its variant blood supply and collateral circulation. Am J Surg 112: 337-347, 1966.

9 Song SY, Chung JW, Lim HG and Park JH: Nonhepatic arteries originating from the hepatic arteries: Angiographic analysis in 250 patients. J Vasc Interv Radiol 17: 461-469, 2006.

10 Camacho JC, Moncayo V, Kokabi N, Reavey HE, Galt JR, Yamada K, Kies DD, Williams RS, Kim HS and Schuster DM: (90)Y Radioembolization: Multimodality imaging pattern approach with angiographic correlation for optimized target therapy delivery. Radiographics 35: 1602-1618, 2015.

11 Lee EW, Thakor AS, Tafti BA and Liu DM: Y90 selective internal radiation therapy. Surg Oncol Clin N Am 24: 167-185, 2015.

12 Salem R, Thurston KG, Covey AM, Brody LA, Maluccio MA, Getrajdman GI and Brown KT: Radioembolization with 90yttrium microspheres: A state-of-the-art brachytherapy treatment for primary and secondary liver malignancies. Part 1: Technical and methodologic considerations. J Vasc Interv Radiol 17: 1251-1278, 2006.

13 Murthy R, Nunez R, Szklaruk J, Erwin W, Madoff DC, Gupta S, Ahrar K, Wallace MJ, Cohen A, Coldwell DM, Kennedy AS and Hicks ME: Yttrium-90 microsphere therapy for hepatic malignancy: devices, indications, technical considerations, and potential complications. Radiographics 25(Suppl 1): S41-S55, 2005.

14 Vente MA, Wondergem M, van der Tweel I, van den Bosch MA, Zonnenberg BA, Lam MG, van Het Schip AD and Nijsen JF: Yttrium-90 microsphere radioembolization for the treatment of liver malignancies: a structured meta-analysis. Eur Radiol 19: 951-959, 2009.

15 Hamoui N, Minocha J, Memon K, Sato K, Ryu R, Salem R and Lewandowski RJ: Prophylactic embolization of the gastroduodenal and right gastric arteries is not routinely necessary before radioembolization with glass microspheres. J Vasc Interv Radiol 24: 1743-1745, 2013.

16 McWilliams JP, Kee ST, Loh CT, Lee EW and Liu DM: Prophylactic embolization of the cystic artery before radioembolization: Feasibility, safety, and outcomes. Cardiovasc Intervent Radiol 34: 786-792, 2011.

17 Salem R, Lewandowski R, Roberts C, Goin J, Thurston K, Abouljoud $\mathrm{M}$ and Courtney A: Use of yttrium-90 glass microspheres (TheraSphere) for the treatment of unresectable hepatocellular carcinoma in patients with portal vein thrombosis. J Vasc Interv Radiol 15: 335-345, 2004.

18 Lee MW, Lee JM, Lee JY, Kim SH, Park EA, Han JK, Kim YJ, Shin KS, Suh KS and Choi BI: Preoperative evaluation of the hepatic vascular anatomy in living liver donors: Comparison of CT angiography and MR angiography. J Magn Reson Imaging 24: 1081-1087, 2006.

19 Laghi A: MDCT Protocols Whole Body and Emergencies. Milan, Springer-Verlag, pp. 96-111, 2012.

20 Saba L: CT Imaging of Hepatic Arteries. In: Computed Tomography-Clinical Applications. InTech Open, pp. 231-240, 2012.

21 Van den Hoven AF, Braat MN2, Prince JF, van Doormaal PJ, van Leeuwen MS, Lam MG, van den Bosch MA: Liver CT for vascular mapping during radioembolisation workup: comparison of an early and late arterial phase protocol. Eur Radiol 27: 6169, 2017. 
22 Tang K, Wang L, Li R, Lin J, Zheng X and Cao G: Effect of low tube voltage on image quality, radiation dose, and low-contrast detectability at abdominal multidetector CT: Phantom study. J Biomed Biotechnol 2012: 130169, 2012.

23 Quaia E: Comparison between $80 \mathrm{kV}, 100 \mathrm{kV}$ and $120 \mathrm{kV} \mathrm{CT}$ protocols in the assessment of the therapeutic outcome in HCC. Liver Pancreat Sci 1: 1-4, 2016.

24 Ng EH, Chung JB, Klass D, Ho SG, Patel R, Chou FY, Legiehn GM and Liu DM: Optimized computed tomographic angiography vessel evaluation protocol (OCTAVE) prior to transarterial radioembolization. Intervent Oncol 360(4): E183-E193, 2016.

25 Price M, Patino M and Sahani D: Computed tomography angiography of the hepatic, pancreatic, and splenic circulation. Radiol Clin North Am 54: 55-70, 2016.

26 Kumamaru KK, Hoppel BE, Mather RT and Rybicki FJ: CT Angiography: Current technology and clinical use. Radiol Clin North Am 48: 213-235, 2010.
27 Silveira LA, Silveira FBC and Fazan VPS: Arterial diameter of the celiac trunk and its branches. Anatomical study. Acta Cirúrg Bras 24: 44-47, 2009.

28 Irfan A and Weir G: Hepatic arterial mapping by CT angiography, cone beam CT and digital subtraction angiography. J Liver Res Disord Ther 2: 00020, 2016.

29 Burgmans MC, Too CW, Kao YH, Goh AS, Chow PK, Tan BS, Tay KH and Lo RH: Computed tomography hepatic arteriography has a hepatic falciform artery detection rate that is much higher than that of digital subtraction angiography and 99mTc-MAA SPECT/CT: Implications for planning $90 \mathrm{Y}$ radioembolization? Eur J Radiol 81: 3979-3984, 2012.

Received April 19, 2018

Revised May 14, 2018

Accepted May 16, 2018 